\title{
Effect of upper extremity coordination exercise during standing on the paretic side on balance, gait ability and activities of daily living in persons with stroke
}

\author{
Ju-O Kim ${ }^{a}$, Byoung-Hee Lee ${ }^{b}$ \\ ${ }^{a}$ Department of Physical Therapy, Bethesda Hospital, Suwon, Republic of Korea \\ ${ }^{b}$ Department of Physical Therapy, College of Health Science and Social Welfare, Sahmyook University, Seoul, Republic of Korea
}

Objective: The purpose of this study was to determine the effect of upper extremity coordination exercise (UECE) during standing on the paretic side on balance, gait ability and activities of daily living (ADL) in persons with stroke.

Design: A randomized controlled trial.

Methods: A total of 27 patients with hemiplegic diagnosis after stroke were divided into two groups. Fourteen patients were in the study group and 13 patients were in the control group. The study group received conventional physical therapy and UECE during standing on the paretic side. The control group received conventional physical therapy and simple upper extremity exercise (SUEE). Subjects in both groups were given upper extremity training for 30 minutes per day, five times a week for 4 weeks. Initial evaluation was performed before treatment and reevaluated 4 weeks later to compare the changes of balance, gait ability and ADL (Korean version of modified Barthel index, K-MBI).

Results: Both groups showed a significant effect for balance, gait ability and ADL $(p<0.05)$. In the Independent t-test, between both groups showed a significant effect for balance and gait ability except ADL $(p<0.05)$.

Conclusions: In this paper, we investigated the changes in balance, walking, and ADL through UECE. We found significant changes in the study group and the control group. Results of the present study indicated that UECE during standing on the paretic side for 4 weeks had an effect on balance, gait ability and ADL (K-MBI) in persons with hemiplegia after stroke.

Key Words: Activities of daily living, Balance, Gait, Stroke, Upper extremity coordination exercise

\section{Introduction}

The neurological disorders that may occur in stroke patients include motor and sensory disorders, cognitive disorders, perception disorders, language disorders, and emotional disorders and several patients suffer from permanent disabilities as they do not recover movement and function due to the loss of motor function [1].

Many stroke patients cannot use their impaired upper limb properly despite long-term intensive therapy because damage to the upper limb after stroke is one of the most common and severe stroke side effect [2]. The hand function of the upper limb is the most highly damaged one in brain damaged patients. This is the most abundant cell in the cerebral cortex. It is responsible for the control of hand [3]. Adjustment to a specific pattern is required for segments between the neck and the body in order to maintain the stability of the upper body during walking [4]. Arbitration is particularly required for this adjustment because the upper body segments can affect dynamic stability [5]. Conventional rehabilitation therapy for spasticity administered by therapists includes passive stretching and range of motion exercise treatment. The amount and effects of repetitive exercise manually induced by therapists may differ according to the

Received: 21 March, 2017 Revised: 5 June, 2017 Accepted: 5 June, 2017

Department of Physical Therapy, College of Health Science and Social Welfare, Sahmyook University, 815 Hwarang-ro, Nowon-gu, Seoul 01795 , Republic of Korea Tel: 82-2-3399-1634 Fax: 82-2-3399-1639 E-mail: 3679@syu.ac.kr

(c) This is an Open-Access article distributed under the terms of the Creative Commons Attribution Non-Commercial License (http://creativecommons.org/licens es/by-nc/4.0) which permits unrestricted non-commercial use, distribution, and reproduction in any medium, provided the original work is properly cited.

Copyright $\odot 2017$ Korean Academy of Physical Therapy Rehabilitation Science 
therapists' levels of experience [6]. Despite their participation in standard rehabilitation programs restoration of arm and hand function for activities of daily living (ADL) is not achieved in the majority of stroke patients. In the first weeks and months after stroke, a positive relationship between the dose of therapy and clinically meaningful improvements has been demonstrated [7]. The asymmetric gait cycle of stroke patients also affects their gait symmetry. The speed, balance and the symmetry of gait are correlated with motor recovery, upper-extremity muscle strength, ADL and postural control [8]. In Scena et al. [9], the reliability and validity of the functional reach test (FRT) were evaluated in the balance evaluation of patients with neurological impairment. The goal of rehabilitation is to enhance ADL performance so that patients can eventually carry out ADL independently. Various tools have been used for evaluating the status of stroke patients and the achievement of treatment goals. Barthel index (BI) was revised as modified BI (MBI) to be more conveniently and effectively used all over the world [10]. And the Korean version of MBI (K-MBI) has been developed. Various tools for assessing the upper extremities that play important roles in ADL have also been developed. Loss of upper extremity functions is determined based on the levels of paresis, loss of fractionated movements, abnormal muscle tone, and somatosensory impairments [11,12].
The effects of upper extremity co-ordination exercise (UECE) have been demonstrated in previous studies $[3,9,12]$. These studies were conducted with walking or meaningless upper limb movement. However, the effects of UECE during standing on a paretic side on functional movements of hemiplegic patients after stroke remain unclear. Therefore, the objective of this study was to determine the effects of UECE during standing on a paretic side on activity of daily living, balance and gait ability of stroke patients.

\section{Methods}

\section{Subjects}

This study was conducted at Bethesda Hospital. A total of 27 patients who were admitted to inpatient rehabilitation center were selected as the study group. The inclusion criteria were: those who have been diagnosed with hemiplegia for more than 6 months [13]; those who were able to respond properly from a sensory stimuli given by the examiner with over 24 points in mini mental state examination [14]; those who were able to carry out instructions given by verbal command [15]; those who were independent in ambulation with or without a walking aid [16]; and those who understood the content of study and agreed to participate actively (Figure 1). The study was approved by the Institutional Review

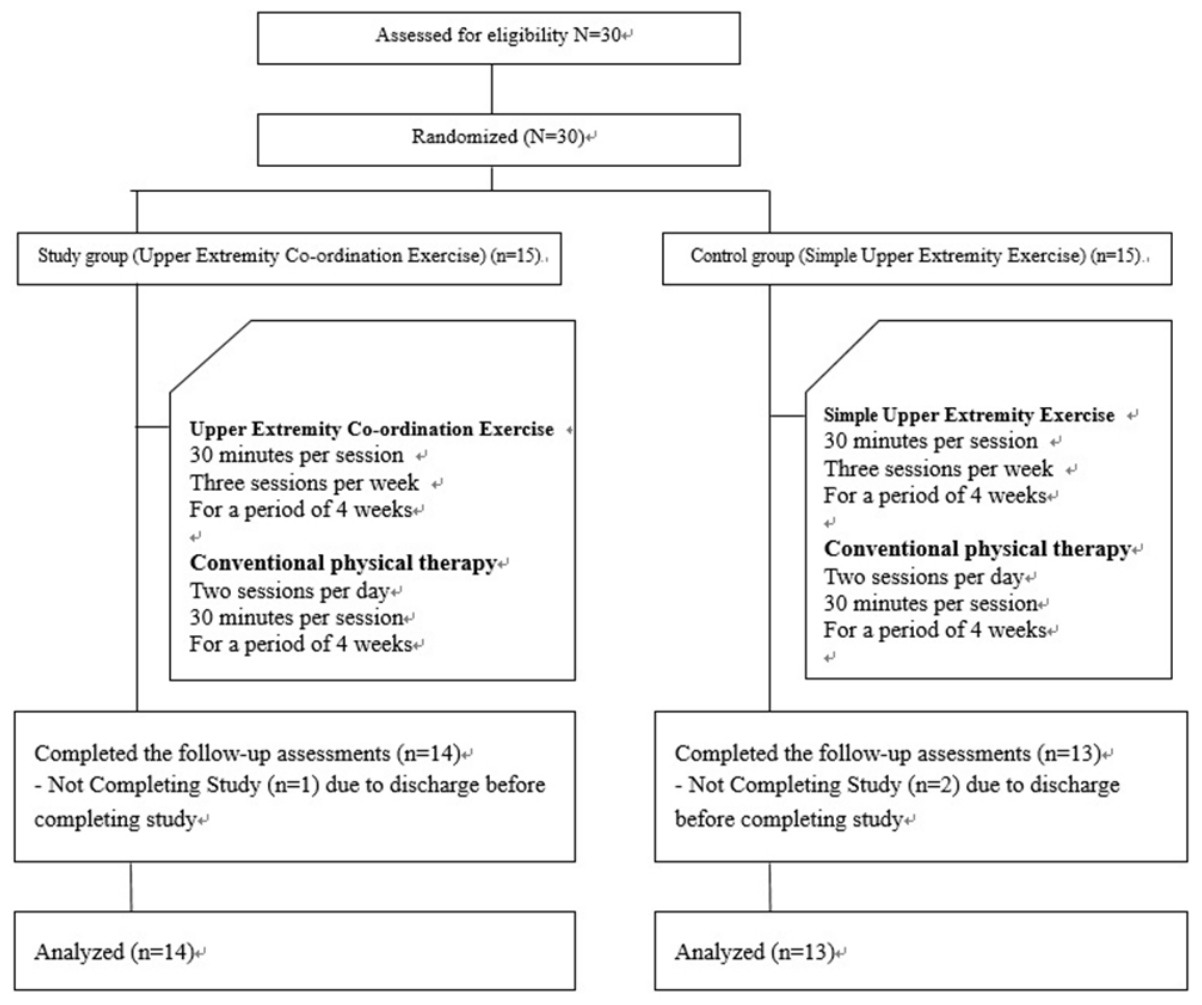

Figure 1. Flow of participants through the exercise program. 
Board of the Sahmyook University (IRB No. 2-1040781AB-N-01-2017047HR).

\section{Experimental procedures}

27 participants were randomly assigned with computer lottery study group (UECE; $\mathrm{n}=14$ ) and control group (simple upper extremity exercise [SUEE]; $n=13$ ). The UECE applied in this study was a revised version of that used in a previous study $[17,18]$. Generally, upper limb exercise methods did not emphasize the stabilization of the shoulder, and the compensatory action of the opposite side occurred. In this paper, we apply the exercise method by revising and revising the previous study. All operations are performed basi- cally after stabilization of the line ridge by attaching the scapula inward. When performing all the operations, the therapist intervenes and exercises while adjusting the compensatory action of the opposite side (Figure 2).

Briefly, participants received 30 minutes of strengthening exercise on UECE in standing posture. Exercise was conducted 3 sessions per week for a total of 4 weeks. Participants in the Control group (SUEE) received $30 \mathrm{mi}-$ nutes of shoulder movement exercise (flexion, extension, abduction, adduction, internal rotation, external rotation) in standing posture. All operations are simple, without special constraints, and allow compensation on the other side. All the participants in both groups received 30 minutes per ses-
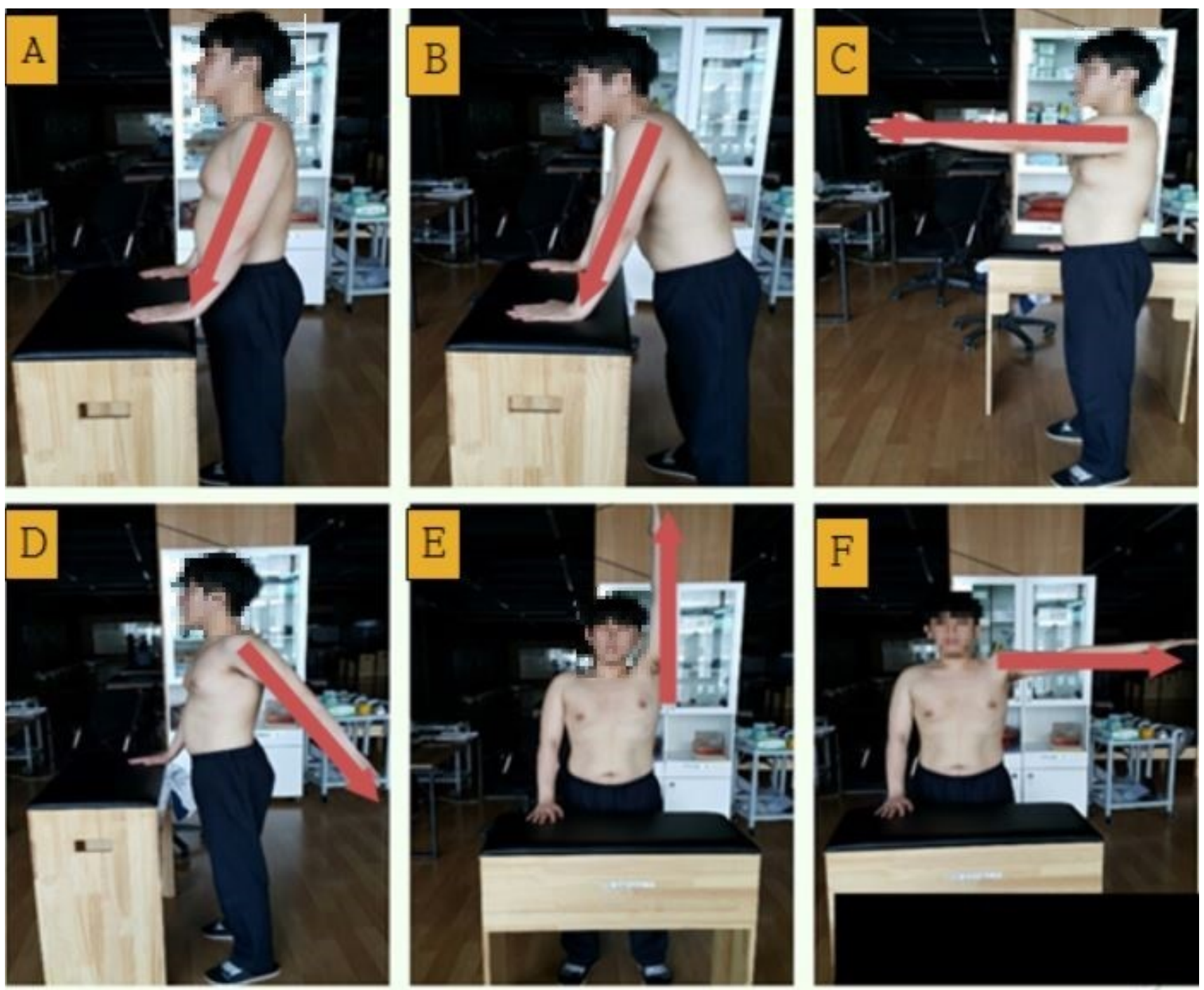

Figure 2. Upper extremity co-ordination exercise. Red arrow means affected side. (A) Bring the scapula to the center and place both hands on the table. (B) Supporting weight on the table. (C) Shoulder protraction of affected side upper limb. (D) Shoulder extension of affected side upper limb. (E) Shoulder flexion of affected side upper. (F) Shoulder abduction of affected side upper limb. 
sions of conventional physical therapy for 5 sessions per week for 4 weeks. The main reasons for 3 drop-outs from the study were a personal issue.

\section{Outcome measurement}

\section{Timed up and go test}

Timed up and go test (TUG) is functional flexibility test invented by Podsiadlo and Richardson [19]. It was designed as a quick measure of basic balance and mobility skill of elderly people. The time taken for subjects to rise from an armchair, walk 3 meters, turn, and return to the chair is measured in seconds.

\section{Functional reach test}

As above mentioned, the FRT is based on analyzing the limits of anterior-posterior stability in an upright position, in the absence of external perturbations. It assesses the maximum forward displacement that a subject can reach without losing balance [9].

\section{Korean version of modified Barthel index}

Various tools have been used for evaluating the status of stroke patients and the achievement of treatment goals. BI was revised as MBI to be more conveniently and effectively used all over the world. And the K-MBI has been developed $[10,11]$.

\section{Statistical analysis}

All the variables are presented as mean and standard deviation, and the statistical analysis was conducted using IBM SPSS Statistics ver. 19.0 (IBM Co., Armonk, NY, USA). To assess the general characteristics of the subjects, descriptive statistics was used. Paired t-test used to define the differences between pretest and posttest in each group, and in- dependent t-test was used to define the differences between study group and control groups after 4 weeks of training. Statistical significance was considered when $p$-value was less than 0.05 .

\section{Results}

This study enrolled 27 patients, 14 patients in the study group and 13 patients in the control group, and analyzed intervention effects with both paired t-test and independent $\mathrm{t}$-test. Results are presented in Table 1. Study group was a significant effect for TUG ( $\mathrm{t}=6.98, p=0.000)$, FRT affected (FRTA $[\mathrm{t}=-3.72, p=0.003]$ ), FRT non-affected (FRTN [ $\mathrm{t}=$ $-4.37, p=0.001])$, and K-MBI $(\mathrm{t}=-5.40, p=0.000)$. Control group was a significant effect for TUG $(\mathrm{t}=4.66, p=0.001)$, FRTA $(\mathrm{t}=-2.92, p=0.013), \operatorname{FRTN}(\mathrm{t}=-2.92, p=0.013)$, and $\mathrm{K}$-MBI $(\mathrm{t}=-2.88, p=0.014)$. Both groups improved their ability to gait, balance and ADL. This confirms that both UECE and SUEE have improved function through upper extremity exercise. There was a significant difference between the two groups in the independent t-test. The study group exhibited significantly greater improvement than the control group in the TUG $(\mathrm{t}=-0.571, p=0.023)$, FRTA $(\mathrm{t}=-0.222$, $p=0.031)$, and FRTN $(\mathrm{t}=-0.700, p=0.012)$, whereas no significant difference was found between the groups for the K-MBI score $(\mathrm{t}=1.297, p=0.081$; Table 1$)$.

\section{Discussion}

UECE is particularly affected by scapular stability during movement in sagittal \& coronal planes. Previous studies on recovery of upper extremity function have investigated the effects of balance, upper-limb function, and gait speed in post-stroke patients $[3,9,12]$.

Table 1. Comparison of upper extremity co-ordination exercise on activity daily living, balance and gait ability

$(\mathrm{N}=27)$

\begin{tabular}{|c|c|c|c|c|c|c|c|}
\hline \multirow{2}{*}{ Variable } & \multicolumn{3}{|c|}{ Study group $(\mathrm{n}=14)$} & \multicolumn{3}{|c|}{ Control group $(n=13)$} & \multirow{2}{*}{$\mathrm{t}(p)^{\mathrm{b}}$} \\
\hline & Pre-test & Post-test & $\mathrm{t}(p)^{\mathrm{a}}$ & Pre-test & Post-test & $\mathrm{t}(p)^{\mathrm{a}}$ & \\
\hline TUG (s) & $12.15(3.32)$ & $10.60(2.83)$ & $6.98(0.000)^{\mathrm{c}}$ & $12.84(2.87)$ & $11.82(2.73)$ & $4.66(0.001)^{\mathrm{c}}$ & $-0.571(0.023)^{\mathrm{c}}$ \\
\hline FRTA $(\mathrm{cm})$ & $18.57(8.01)$ & $20.07(8.10)$ & $-3.72(0.003)^{c}$ & $19.69(5.26)$ & $20.38(5.18)$ & $-2.92(0.013)^{\mathrm{c}}$ & $-0.222(0.031)^{\mathrm{c}}$ \\
\hline FRTN $(\mathrm{cm})$ & $17.00(6.96)$ & $18.43(6.65)$ & $-4.37(0.001)^{\mathrm{c}}$ & $18.08(7.00)$ & $18.77(6.96)$ & $-2.92(0.013)^{c}$ & $-0.700(0.012)^{\mathrm{c}}$ \\
\hline K-MBI (score) & $75.57(11.85)$ & $79.14(13.24)$ & $-5.40(0.000)^{\mathrm{c}}$ & $70.00(10.32)$ & $71.23(11.27)$ & $-2.88(0.014)^{c}$ & $1.297(0.081)$ \\
\hline
\end{tabular}

Values are presented as mean (SD).

TUG: timed up and go test, FRTA: functional reach test affected, FRTN: functional reach test non-affected, K-MBI: Korean version of modified Barthel index.

${ }^{\mathrm{a}}$ Paired t-test, ${ }^{\mathrm{b}}$ Independent t-test, ${ }^{\mathrm{c}}$ Statistics significant difference between groups $(p<0.05)$. 
Movement disorders can bring about limitations to muscle control and motion functions or mobility. They can also result in degradation of balance control abilities. Hemiplegia is accompanied by increased posture instability, asymmetrical weight bearing, impairment of body weight transfer capabilities, and decreased postural stability [20,21]

According to Eng and Chu [22], gait abilities of paretic and non-paretic lower limbs are not identical. In addition, the paretic side is less competent in weight bearing ability. In particular, slow gait velocity and reduced gait endurance may cause many difficulties in the recovery of gait ability. Overcoming these difficulties is a crucial objective of stroke patient rehabilitation [23]. Many studies have reported treatment methods for improving the gait ability of stroke patients [3]. Stroke patients suffer from deficits in functional ambulation capacity, balance, walking velocity, cadence, stride length, temporal gait pattern, and muscular activity pattern. Generally, stroke patients show decreased walking velocity, which can result in decreased paretic stance phase but increased paretic swing phase [24].

According to Hill et al. [25], gait speed and shoulder girdle strength are improved $(p<0.05)$ after 4 weeks of training for stroke patients and in the study group of this study, gait speed (TUG test) was changed from 12.15 seconds to 10.60 seconds. Upper extremity hemiparesis can impair the performance of many daily activities such as dressing, bathing, self-care, and writing, thereby resulting in reduced functional independence. Therefore, stroke survivors need to participate in rehabilitation programs to achieve functional independence [1]. Hemiparesis of the upper extremity is often the most severe one in the most distal regions. Although proximal muscles and joints may be the least affected ones, purposeful movement requiring precise control of the proximal segments is slow, inaccurate, and poorly coordinated [26]. Approximately one third of all stroke survivors have significant residual disability. The severity of upper extremity hemiparesis is a significant determinant of post-stroke disability and quality of life [27].

Postural control of the trunk is also a critical component of independent performance of ADL. Hsieh et al. [28] have investigated the relationship between trunk control and comprehensive ADL. A paretic arm can change body orientation because shoulder girdle stabilizers are often so impaired by muscle weakness that they cannot overcome arm weight or maintain anatomical characteristics. Weakness of scapular stabilizers has been shown to increase motor impairment of upper extremities and consequently affect many stroke sur- vivors to achieve independent daily livings [29]. The efficacy of UECE in improving balance and performance of daily activities by stroke patients was also investigated in this study. In the study group of this study, FRTA was changed from 18.57 to $20.07 \mathrm{~cm}$. An important finding of this study was a statistically significant improvement in the function of the paretic upper extremity after the UECE. This means that balance ability of the study group is improved. Also, in the study group, K-MBI was improved from 75.57 to 79.14 points.

Previous studies evaluated functional changes on ADL [10]. In this paper, we investigated the changes in ADL through balance enhancement due to changes in upper limb function, and found significant changes in the study group using K-MBI.

In this paper, we investigated the changes in balance, walking, and ADL through UECE. We found significant changes in the study group and the control group.

Results of the present study indicated that UECE during standing on a paretic side for 4 weeks had an effect on the Balance, gait ability and ADL (K-MBI) of hemiplegic patients after stroke.

In future studies, more efficient research should be done to improve the balance, gait ability and activity of daily living of stroke patients by revising and supplementing the contents of this paper.

\section{Conflict of Interest}

The authors declared no potential conflicts of interest with respect to the authorship and/or publication of this article.

\section{References}

1. Trombly CA, Wu CY. Effect of rehabilitation tasks on organization of movement after stroke. Am J Occup Ther 1999;53: 333-44.

2. Woodson C, Bandy WD, Curis D, Baldwin D. Relationship of isokinetic peak torque with work and power for ankle plantar flexion and dorsiflexion. J Orthop Sports Phys Ther 1995;22: 113-5.

3. Stephenson JL, Lamontagne A, De Serres SJ. The coordination of upper and lower limb movements during gait in healthy and stroke individuals. Gait Posture 2009;29:11-6.

4. Cromwell RL, Aadland-Monahan TK, Nelson AT, SternSylvestre SM, Seder B. Sagittal plane analysis of head, neck, and trunk kinematics and electromyographic activity during locomotion. J Orthop Sports Phys Ther 2001;31:255-62.

5. Winter DA, Patla AE, Frank JS, Walt SE. Biomechanical walk- 
ing pattern changes in the fit and healthy elderly. Phys Ther 1990;70:340-7.

6. Selles RW, Li X, Lin F, Chung SG, Roth EJ, Zhang LQ. Feedback-controlled and programmed stretching of the ankle plantarflexors and dorsiflexors in stroke: effects of a 4-week intervention program. Arch Phys Med Rehabil 2005;86:2330-6.

7. Jørgensen HS, Nakayama H, Raaschou HO, Olsen TS. Stroke. Neurologic and functional recovery the Copenhagen Stroke Study. Phys Med Rehabil Clin N Am 1999;10:887-906.

8. Hendrickson J, Patterson KK, Inness EL, McIlroy WE, Mansfield A. Relationship between asymmetry of quiet standing balance control and walking post-stroke. Gait Posture 2014;39: 177-81.

9. Scena S, Steindler R, Ceci M, Zuccaro SM, Carmeli E. Computerized functional reach test to measure balance stability in elderly patients with neurological disorders. J Clin Med Res 2016; 8:715-20.

10. Shah S, Vanclay F, Cooper B. Improving the sensitivity of the Barthel index for stroke rehabilitation. J Clin Epidemiol 1989; 42:703-9.

11. Jang SH, Rah UW, Kim YC, Park YS, Jo D, Kim YC; Korean Academy of Medical Sciences. Development of Korean academy of medical sciences guideline-rating the impairment in pain. J Korean Med Sci 2009;24 Suppl 2:S330-7.

12. Heller A, Wade DT, Wood VA, Sunderland A, Hewer RL, Ward E. Arm function after stroke: measurement and recovery over the first three months. J Neurol Neurosurg Psychiatry 1987;50:714-9.

13. Patterson SL, Forrester LW, Rodgers MM, Ryan AS, Ivey FM, Sorkin JD, et al. Determinants of walking function after stroke: differences by deficit severity. Arch Phys Med Rehabil 2007;88: 115-9.

14. Dunsky A, Dickstein R, Marcovitz E, Levy S, Deutsch JE. Home-based motor imagery training for gait rehabilitation of people with chronic poststroke hemiparesis. Arch Phys Med Rehabil 2008;89:1580-8.

15. Lin JJ, Hanten WP, Olson SL, Roddey TS, Soto-quijano DA, Lim HK, et al. Functional activity characteristics of individuals with shoulder dysfunctions. J Electromyogr Kinesiol 2005;15: 576-86.

16. Pang MY, Eng JJ, Dawson AS. Relationship between ambulatory capacity and cardiorespiratory fitness in chronic stroke: influence of stroke-specific impairments. Chest 2005;127:495-501.

17. Ada L, Dorsch S, Canning CG. Strengthening interventions in- crease strength and improve activity after stroke: a systematic review. Aust J Physiother 2006;52:241-8.

18. Awad A, Shaker H, Shendy W, Fahmy M. Effect of shoulder girdle strengthening on trunk alignment in patients with stroke. J Phys Ther Sci 2015;27:2195-200.

19. Podsiadlo D, Richardson S. The timed "Up \& Go": a test of basic functional mobility for frail elderly persons. J Am Geriatr Soc 1991;39:142-8

20. Horstmann S, Koziol JA, Martinez-Torres F, Nagel S, Gardner $\mathrm{H}$, Wagner S. Sonographic monitoring of mass effect in stroke patients treated with hypothermia. Correlation with intracranial pressure and matrix metalloproteinase 2 and 9 expression. J Neurol Sci 2009;276:75-8.

21. Park JH, Hwangbo G, Kim JS. The effect of treadmill-based incremental leg weight loading training on the balance of stroke patients. J Phys Ther Sci 2014;26:235-7.

22. Eng JJ, Chu KS. Reliability and comparison of weight-bearing ability during standing tasks for individuals with chronic stroke. Arch Phys Med Rehabil 2002;83:1138-44.

23. Jette AM. Toward a common language for function, disability, and health. Phys Ther 2006;86:726-34.

24. Patterson SL, Rodgers MM, Macko RF, Forrester LW. Effect of treadmill exercise training on spatial and temporal gait parameters in subjects with chronic stroke: a preliminary report. J Rehabil Res Dev 2008;45:221-8.

25. Hill TR, Gjellesvik TI, Moen PM, Tørhaug T, Fimland MS, Helgerud J, et al. Maximal strength training enhances strength and functional performance in chronic stroke survivors. Am J Phys Med Rehabil 2012;91:393-400.

26. Oujamaa L, Relave I, Froger J, Mottet D, Pelissier JY. Rehabilitation of arm function after stroke. Literature review. Ann Phys Rehabil Med 2009;52:269-93.

27. Nakayama H, Jørgensen HS, Raaschou HO, Olsen TS. Compensation in recovery of upper extremity function after stroke: the Copenhagen stroke study. Arch Phys Med Rehabil 1994;75: 852-7.

28. Hsieh CL, Sheu CF, Hsueh IP, Wang CH. Trunk control as an early predictor of comprehensive activities of daily living function in stroke patients. Stroke 2002;33:2626-30.

29. Hardwick DD, Lang CE. Scapular and humeral movement patterns of people with stroke during range-of-motion exercises. J Neurol Phys Ther 2011;35:18-25. 